\title{
A system for supply chain management of covid-19 vaccines using blockchain and bluetooth beacons network based traceability and verification.
}

\author{
Geetansh Atrey ${ }^{1}$, Junali Jasmine Jena ${ }^{1 *}$, Biswa Ranjan Acharya ${ }^{1}$, \\ ${ }^{1}$ School of Computer Engineering, KIIT Deemed to be University, Bhubaneswar-751024, India
}

\begin{abstract}
Ever since a novel Coronavirus disease (COVID-19) emerged in Wuhan(China), things have changed to a large extent. It has shattered the entire world in all the possible sectors by its continuous spread. Scientists all around the world have put in in-numerous amount of efforts as a result of which the world has finally witnessed its some of the first potential vaccines which have got approvals for emergency use in various countries. Now the next crucial step that comes in this process of fighting this virus is the supply of vaccines to all the different parts of the world. But there are certain challenges to this process as vaccines need to be constantly monitored all the time for their temperatures, humidity and other environmental factors which vary a lot depending on the geographical conditions. Also, maintaining drug safety is the most important challenge as counterfeiting of vaccines and drugs has always been a concern for the pharmaceutical industry. Manual traceability for all the ingredients used during manufacturing is a very difficult process and doesn't even give a full guarantee for the same. This can lead to serious health issues in the consumers and can even result in death. The power of Blockchain and it's principle of proof of concept can deal with this problem by maintaining a record for all the events taking place in this process right from manufacturing to vaccinations in hospitals. BLE Beacons can also come very handy here as they are small, inexpensive and easy to use devices that can provide real time updates by tracking various parameters of the vaccine. This paper aims at suggesting an entire system of supply chain of vaccines using Blockchain based record, QR(Quick Response) Codes for vaccine safety and BLE Beacons network for real time tracking and news updates of the vaccines.
\end{abstract}

\section{Introduction}

A novel Coronavirus disease (COVID-19) came into existence when the first case related to this disease came across in Wuhan(China) in December, 2019 as mentioned in [1]. It was identified to be caused by SARS-CoV-2 (Severe acute respiratory syndrome coronavirus 2). As it was found to be a contagious disease, ever since then the world has been constantly battling this virus which led to an ongoing pandemic. It is a newly discovered coronavirus i.e. there was not much research done on this coronavirus, and its potential effect on humans in the future. Thus the challenges were bigger than ever. The most basic challenge was to first being able to detect the presence of virus in humans through a testing kit. Testing is the most crucial step while fighting this virus, as it could not only help in detecting the presence of virus in people but also help to stop its spread. Testing kit also had to be relatively affordable as it was required by every country, right from the most developed to the under-developed ones. As a result of immense research and urgent need, three kinds of testing mentioned in [2] emerged namely : Serological testing (Testing of antibodies presence in blood stream), Isothermal nucleic acid amplification testing (Commonly known as Rapid Tests. Done by extracting the viral RNA and later amplifying it) and Real Time Polymerase chain reaction (RTPCR) testing
(Detecting a specific genetic material in the virus and then trying to match it). This was a major breakthrough as the first hurdle to fight the virus was conquered. Results of testing could help in analyzing the situation of different areas accordingly. Now the next major challenge that the world is facing is the need for the development of vaccine fighting against COVID-19 and thus making people immune to it.

Ever since the emergence of this coronavirus, many institutes and organizations started to take the charge of developing a potential vaccine. A number of initiatives were organized in order to plan and carefully monitor the process of vaccine development and vaccination, the biggest initiative being the COVAX Global Vaccines Facility. In-numerous amount of efforts are being put in development of vaccines all throughout the world. According to WHO in $[3,4]$, over the course of $6-8$ months about 172 countries have engaged with the COVAX facility which is aiming at delivering about 2 billion doses by the end of year 2021. Thus there have been several breakthroughs in the process of development as a number of vaccines have already completed the three phase trials and have been given approval for emergency use. According to RAPS.org in [5], there are 7 vaccines till now which have been authorized or approved, different ones by different countries and about 55 vaccines which are under one of the trial phases. Considering the USA, there are two 
vaccines which have got an approval for emergency use namely, BNT162b2 (Developed by Pfizer, BioNTech \& Fosun Pharma. It is a mRNA based vaccine) and mRNA-1273 (Developed by Moderna, BARDA and NIAID. It is a mRNA-based vaccine). These vaccines are a major breakthrough in controlling the spread of this coronavirus. But to do so, there are certain challenges and hurdles that comes in the way .

First of them being the maintenance of proper environmental conditions required for the vaccine to sustain. According to Pfizer in [6], their BNT162b2 vaccine requires about $70^{\circ} \mathrm{C} \pm 10^{\circ} \mathrm{C}$ and can remain stable for up to 10 days if kept unopened, while on the other hand vaccine by Moderna i.e. mRNA-1273 requires a temperature of $2^{\circ} \mathrm{C}$ to $8^{\circ} \mathrm{C}$ to survive and can remain stable for up to 30 days if kept unopened. Thus there is an important need to constantly monitor these vaccines during all the different processes involved during the supply of these vaccines, right from manufacturing them and storing them in warehouses to delivering the vaccines through different travel methods and vaccinating the patients.

The second biggest challenge that is faced at this stage is fighting against the production of fake vaccines i.e. counterfeiting of vaccines. Pharmaceutical industry has been highly affected by this problem since a long time. In 2009 as mentioned in [7], 20 million tablets, bottles and sachets of counterfeit and illicit narcotics were confiscated in a five-month operation organised throughout China by the International Criminal Police Organization (Interpol) and seven of its South-East Asian neighbours, according to the WHO in [7]. Fake vaccines can lead to serious allergies and health problems in the patients which can even lead to death in the worst case.

Maintaining a proper database of the patients is also a big challenge in this process. Also, spreading right information among the citizens and making them aware about the vaccination schedule is also very important as it can lead to chaos at hospitals/vaccination centers.

A probable solution for all these problems is the use of manpower throughout the vaccination process but it is very ineffective when it comes to large scale. The usage of Blockchain based data record for the patients/consumers of vaccines and maintaining a blockchain within all the different entities of the supply chain can lead to proof of concept and can help to identify malicious participants. Also the usage of BLE (Bluetooth Low Energy) Beacons, which are small, inexpensive Bluetooth devices for real time tracking can help to maintain the environmental conditions needed for different vaccines accordingly.

\section{Supply Chain in Pharmaceuticals : Challenges}

For any product to be successful commercially and to maintain product quality, the supply chain of the product plays the most vital role. Supply chains need to be carefully planned to utilize the maximum potential of a product. The entities involved in a supply chain depends on the various used cases. In the context of medical/pharmaceutical industry, a supply chain generally consists of four entities which are namely Manufacturers, Distributors, Hospitals, and finally the Patients/Consumers.

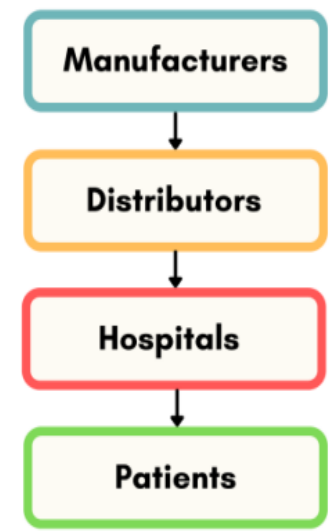

Fig. 1. Entities involved in Supply Chain of Vaccines

\subsection{Manufacturers}

Manufacturing is the process of production and development of finished products right from raw materials to packaging. Group of people or registered companies involved in manufacturing are called manufacturers. Manufacturing is an essential part of Supply Chain Management as they are responsible for creating and producing the goods in large amounts or in bulk. As shown in Fig.1, after the completion of manufacturing, the products are then sent to distributors/wholesalers for further selling. This responsibility comes with a lot of challenges.

Manufacturers need to take care of all the complexities of the raw materials needed for production. In the context of pharmaceutical industry, the raw materials required are immense and are of different chemical natures. Thus importing them with proper care is the first challenge. Manufacturers have to make sure that the storage facilities are properly maintained as different raw materials require different environmental conditions to sustain. The entire process of producing the goods needs to be automated as manual involvement gives a possibility of errors.

Summarizing the challenges faced by the manufacturers, the biggest challenge they face is the need of constant monitoring right from raw materials, to intermediate products to the final product. Manual monitoring is not feasible, plus it also leads to errors. In the context of medical products and vaccines, any kind of altercation in the process of manufacturing at any stage can lead to serious health issues in the consumers/patients and can even lead to death. Thus there needs to be a proper monitoring system, which has high level of accuracy as well as is cost effective.

\subsection{Distributors}

The process of distribution can be defined as the process of delivering the final product manufactured to the right customers. The authorized companies involved in 
distributing are known as Distributors. Distributors play a very vital role in the process of supply chain as they are responsible of selling and delivering the product to customers in general, irrespective of their geographical locations. But again, the process of distribution also faces some critical challenges which need to be dealt properly. As shown in Fig.1, in the context of vaccines the distributors sell the vaccines to the authorized hospitals who are responsible for vaccinating the citizens.

Distributors need to make sure that the products are delivered in time to the next holders. Any kind of delay in the process of delivering might lead to damage or depreciation of the product. Distributors need to make sure that if there are any environmental conditions such as temperatures, air pressures, etc. Required for the product to sustain during transit. In case of COVID-19 vaccines, temperature is one of the parameters which needs to be constantly maintained. Distributors need to make sure that there workers are working in a safe environment. The manual involvement should be made minimal in order to avoid any chaos.

\subsection{Hospitals}

In case of pharmaceutical industry and the process of supply of vaccines, hospitals or vaccination centers are the next stop. Hospitals are responsible for vaccinating the patients/customers. There is a huge network of hospitals, each of which are assigned a particular geographical area for vaccination. Hospitals also face a lot of challenges in the entire process.

Hospitals need to make sure that the entire process of vaccination is done properly with the right methodology. Hospitals need to make sure that there is no chaos of patients coming together because it can lead to further spread of the virus. Hospitals need to make sure that the directions inside them are clear enough for the patients to understand and they reach the rightly allotted rooms without facing any difficulty. Hospitals complete the process of vaccination by vaccinating the patients according to the guidelines provided to them by the government.

\subsection{Patients/Consumers}

Consumers are the final holders and the users of any supply chain product. In the context of vaccination, the consumers are the patients which are vaccinated. They need to properly made aware about the details of the vaccine and the entire process of vaccination. Prior to that their data also has to be collected and they need to be properly informed about the schedule of the vaccination. They also need to be made aware about the hospitals/vaccination centers they need to go to get vaccinated.

Overall there are some major challenges that the entire supply chain system faces right from manufacturers to the consumers, which need to be dealt very effectively and swiftly. A possible solution for the problems is the usage of Blockchain for maintaining a database of every event/transaction that takes place on a bottle of vaccine. The usage of Bluetooth based Beacons can solve the issue of monitoring the vaccines during different stages and also the issue of informing the patients about the vaccines, the hospitals and even the indoor navigation of the hospitals.

\section{Possible Solutions}

\subsection{Blockchain : Concepts and Application in Pharmaceutical Supply Chain Management}

Blockchain is referred to as the technology in which a database of chain of blocks is maintained, linked together and backed by cryptography. The credit of inventing this technology goes back to 2008, by a person (or a group of person) with the name of Satoshi Nakamoto who invented Bitcoins. The transaction ledger of Bitcoins which was to be kept public was made using Blockchain technology. There are a lot of features associated with Blockchain which make the technology very effcient and secure.

Blockchain is an increasing list of records (can be public or private) that are connected by cryptography, called blocks. It follows the principle of decentralization. Each block includes the previous block's cryptographic hash, a time stamp, and transaction data. Here the data is generally maintained using Merkle Tree. Because of this type of design, any kind of altercation or tampering in the data is practically not possible on a blockchain because in doing so, the data in the subsequent blocks would also change and thus an inconsistency would easily be reflected. A blockchain is usually operated for use as a distributed ledger done by a peer-to-peer network. This peer-to-peer network collectively agrees to a protocol for communication internally within the network and also for validating the blocks that are been mined by the miners for them to be attached to the blockchain.

The creation of the Ethereum platform in 2017 (by Vitalik Buterin) signalled a significant shift in blockchain technology, as it was now able to move beyond its financial limitations. Developers can now create decentralised applications using the principle of smart contracts.

Nick Szabo, a computer scientist and cryptographer, invented the term in 1996. Smart contracts, according to Szabo, can be implemented using a public ledger. An automated computerised protocol for digitally facilitating, confirming, or implementing the negotiation or performance of a legal contract by bypassing intermediaries and validating the contract directly over a decentralised network - quicker, cheaper, and more safe. Blockchain has the potential to be a game-changing tool for smart contract implementation.

Smart contracts provide some of the most significant benefits that Blockchain 1.0 (Bitcoin Revolution) does not. First of all comes the Immutability. Once the contract has been fixed and written to the public ledger, no party will be able to amend it (the Blockchain). Another is the Distributed network. Any involved party can verify all steps of the contract; no one can later argue that the contract was not validated. 
Blockchain are of different types depending on the use-cases and requirements. Although there are no limit to which blockchain can be modified but in general there are 3 common types of blockchain which are as follows :

\subsubsection{Public Blockchain}

A public blockchain has no limitations on the participants to become a part of the blockchain. There are no fixed participants as anyone having internet and capable of sending and verifying transactions can join the peer-to-peer network.

\subsubsection{Private Blockchain}

Permissioned types of blockchain are known as private blockchain. In this type of network, only restricted and authorized members can join that too only after an invitation from the system administration. Although these type of blockchain go against the idea of decentralization, but in case of corporate use these type of blockchain are popular and feasible to use.

\subsubsection{Hybrid Blockchain}

There is a mixture of centralized and decentralized elements in a hybrid blockchain. The precise operations of the network will differ depending on which aspects of the decentralisation of centralization are utilized. full and complete as possible and include the country.

A block is a container data structure in the blockchain that comprises a sequence of transactions. The header and the data (transactions) are the two parts of the Block. A block's header binds the transactions; any change in a transaction causes a change in the block header. The headers of subsequent blocks are linked in a chain; if you want to make any changes, you must update the entire blockchain.

Mining is very important in order to take these blocks into account by applying them to the blockchain. Blockchain mining is a peer-to-peer computer method that is used to protect and validate transactions. Blockchain miners are those that link transaction data to the Blockchain (global public ledger of past transactions). In the ledgers, blocks are secured by Blockchain miners and are connected to each other forming a chain. The mining ensures that no node has the power to sabotage the network and gain control .No one can hold the control of the cryptocurrency. Blockchain miners secure blocks in the ledgers, which are then linked together to form a chain. Mining means that no node has the ability to take control of the network and sabotage it. The cryptocurrency cannot be regulated by anyone. The nodes' computational effort in reaching consensus would be compensated with cryptocurrency created and controlled by the network. Blockchain ensures that the currency is secure and tamper-proof.

Using blockchain in the supply chain management of vaccines can immensely solve a lot of problems and can tackle a lot of challenges faced during different stages. The fight against the fake vaccines and the counterfeiting of vaccines can be solved very effectively using blockchain. Every transaction/event occurring at any stage can be recorded on a decentralized database that is blockchain. The best feature here is that this database is tamper-proof. Now in the future at any stage, if the vaccine is found to be fake then the blockchain can be checked for identifying the particular manufacturer / distributor / hospital involved in these malicious and illegal affairs. Also the database of citizens required for the consensus details can be stored on a blockchain which is kept private and only accessed by authorized personnel. This makes the data of the citizens secure and due to its tamper-proof and duplicate identifying nature, a very precise and effective database can be made which can be used by the manufacturers to check for the supply accordingly. An overall system of hybrid blockchain with multiple internal private blockchain can solve this issue to a large extent.

\subsection{BLE Beacons : Concepts and Application in Pharmaceutical Supply Chain Management}

BLE Beacon or Bluetooth Low Energy Beacon often marketed as Bluetooth Smart is a device which works on the principle of Bluetooth Low Energy which is a form of wireless communication intended for short-range communication in particular. In the way that it helps devices to connect with one another, BLE has similar characteristics as a WiFi. But there are distinct use-cases where BLE is a preferred form of communication instead of $\mathrm{WiFi}$, especially in those types of communication where battery requirement is more emphasized over the fast rate of communication and data transfers.

Bluetooth Beacons are helpful while transmitting and broadcasting signals to nearby (generally the range for BLE beacons is from 20-30 meters) devices. When in near proximity to a beacon, the hardware helps smartphones, tablets and other gadgets to perform acts. They transmit a universal specific identifier picked up by a compatible software or operating system for which Bluetooth beacons use Bluetooth low energy proximity sensing. Nowadays, Bluetooth beacons are generally used in the field of marketing as they can be a good source of information about sales, discounts, and reach to shop owners and can attract a lot of customers with real-time updates.

BLE Beacons can solve a lot of issues and challenges faced by the pharmaceutical supply chain management. The major issue which is faced by entities of the supply chain right from the manufacturers, distributors, to the hospitals was the monitoring of the vaccines for their suitable environmental conditions such as temperature, air pressure, etc for them to sustain. BLE beacons are small and inexpensive and can provide this facility of real-time tracking of the vaccines right from the warehouses to the delivery vans/trucks and even airplanes. BLE beacons in correspondence with a WiFi hotspot can send the data collected in real-time to the cloud database and can alert the caretakers accordingly if any kind of altercation in the behaviour or 
environment of vaccines is observed. It also reduces the use of manpower and along with it provides high rates of accuracy. BLE beacons can also be installed in localities in order to keep the citizens of the country updated with the latest information about COVID-19 and the plan of the government about the vaccination and its schedule, whenever a person is in proximity (2030 meters) with the beacon through the use of Bluetooth Smart and smartphone devices. BLE beacons can also be installed in the hospitals which can help the patients/consumers of the vaccines when they go to a particular hospital for indoor navigation and guiding them to the right rooms allotted to them.

Overall, a system comprising of blockchain based database for recording any events/transactions and a BLE beacon network for monitoring the vaccines and providing updates about them can immensely solve all the issues faced by the pharmaceutical supply chain making it more effective which would lead to a major impact on stopping and controlling the spread of this coronavirus.

\section{Proposed System}

This paper firstly proposes a system architecture which consists of a peer-to-peer network based on a hybrid blockchain which is used for verification of vaccines and maintaining vaccine safety. Firstly a private blockchain, maintained and restricted to the government authorities is maintained for the collection of the data of the citizens of the country who will be vaccinated i.e. the data of the patients/consumers. Secondly, each of the entities of the vaccine supply chain i.e. manufacturers, distributors and the hospitals maintain a private blockchain of their own for any type of event/transaction taking place on their processes respectively. Thirdly, a private blockchain of several private blockchain i.e. a hybrid blockchain is maintained for keeping a check on the requests of

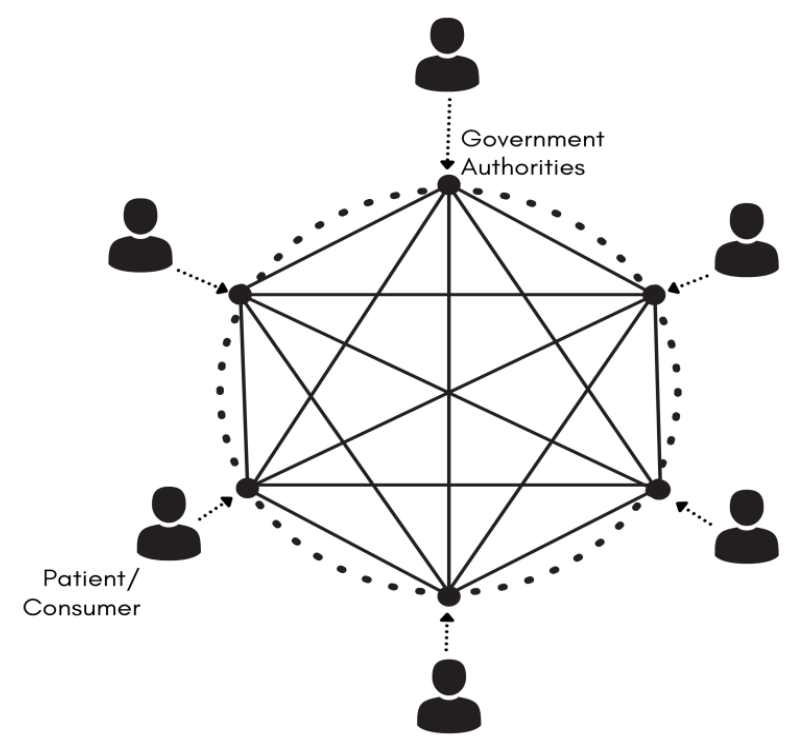

Fig. 2. Private Blockchain for Data Collection of the Citizens for Vaccination

inquiry of the vaccines from the patients. The first reference to slightly similar type of blockchain is mentioned in [8] where the hybrid blockchain is a combination of private chains with public blockchain. The model suggested in this paper consists of the following three type of blockchain :

\subsection{Private Blockchain based collection of citizens data}

Vaccination is a long process which requires a lot of steps, each of which need to be properly taken care of. The first process in the vaccination is the collection of latest data of the citizens who will be vaccinated in order to plan the supply accordingly. This database is maintained on a private blockchain where only the restricted government authorities can enter the blockchain as a participant. The government authorities can collect the data from the consumers by reaching out to them locally and asking for their personal details. These personal details would then be stored on a block inside the block body in an encrypted manner which would make it secure and tamper-proof. That block would later be verified by the entire peer-to-peer network for any duplicacy, or faults and then would be added to the blockchain i.e. the decentralized database after the block is mined. As shown in Fig.2, the citizens are the patients/consumers of the vaccines which are not the part of the blockchain but they submit their required details to the government authorities who encrypt their data and store it. This helps in achieving more efficiency and speed as if all the citizens were made a part of the blockchain and thus making the blockchain would lead to an immense amount of members and nodes on the blockchain which would increase the load on the blockchain and thus slowing the process to a large extent. 


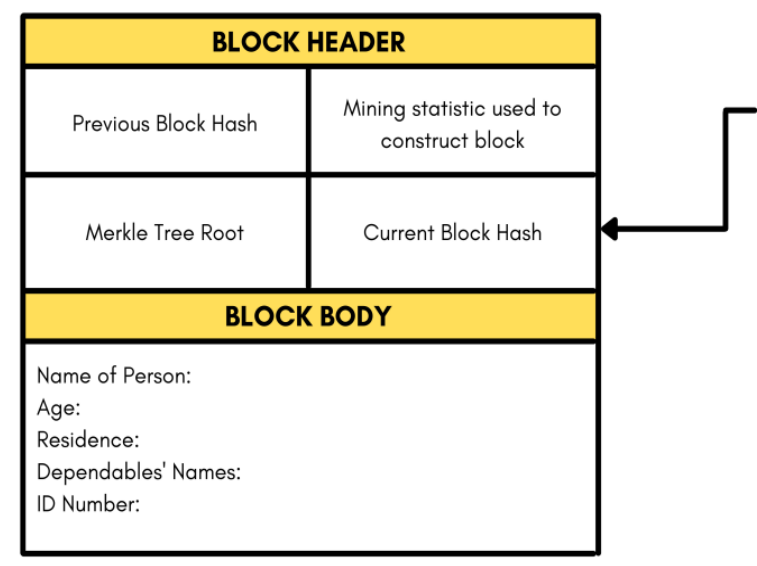

Fig. 3. Block Information for the Citizens Database : consisting of Block Header and Block Body

The block for the Data Collection of the Citizens by the government authorities consists of the block header and the block body. Block header consists of the parameters which are related to block verification. They are namely: Previous Block Hash, Merkle Tree Root, Mining statistic used to construct the block (might be different according to specific use-case), and the Current Block Hash, which is connected to the next block using the block pointed indicated by the arrow sign. The block body consists of the actual information to be stored in the database. These are namely, Name of Person, Age, Residence, Dependables' Names and ID Number (Might be different according to different countries. Should be an unique identity proof). These are stored in the block in an encrypted manner which ensures the safety of the citizens data. During verification, any kind of duplicacy is also checked and thus making it a very effective format of storing.

\subsection{Private Blockchain for any Events or Transactions of Manufacturers, Distributors and Hospitals}

Now the next procedure in solving the problems of supply chain is the maintenance of the database for all the events/transactions happening on manufacturers, distributors and hospitals. Events are referred to as any kind of processes performed on the vaccine. For example, suppose a manufacturer with ID : MFR-1 is involved in the manufacturing of the vaccine with ID : VAC-1. Then when MFR-1 completes the manufacturing of VAC-1, then the transaction would be MFR-1-->VAC-1 and the block body for this transaction would contain the information of the vaccine and the processes and operations performed on it. Similarly, after the distribution is completed by a distributor with ID: DIS-1 on VAC-1 then the distributor transaction would look like DIS-1-->VAC-1. And similarly a hospital with ID: HOS-1 after completing the process of supplying the VAC- 1 to the patients would make a transaction HOS-1-->VAC-1. The block for the manufacturers blockchain contains a block header and a block body. As shown in Fig.4, the block header consists of necessary blockchain parameters i.e. Previous Block Hash, Merkle Tree Root, Mining statistic used to construct the block (might be different according to specific use-case), and the Current Block Hash, which is connected to the next

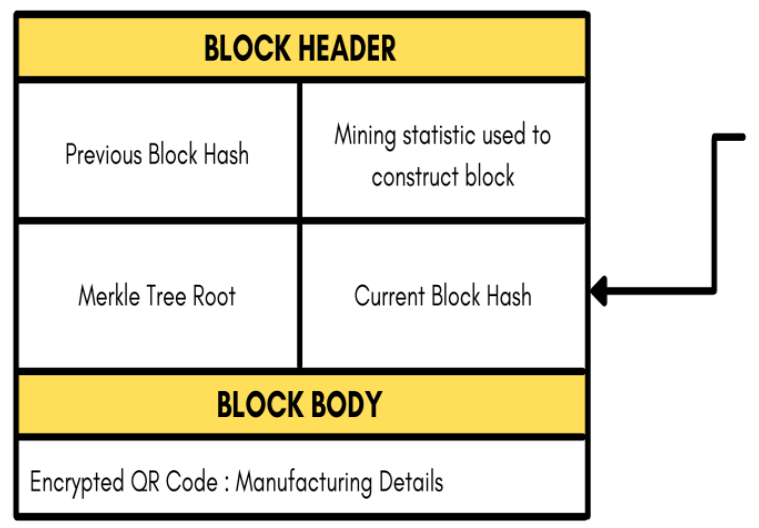

Fig. 4. Block Information for the Manufacturers Database : consisting of Block Header and Block Body

block using the block pointed indicated by the arrow sign. The block body consists of individual information, i.e. an encrypted QR (Quick Response) code, to be stored in the database. This QR code consists of all the manufacturing details of the vaccine, i.e. its ingredients, raw materials, manufacturer details (ID, Name, Address, etc), time-stamps, vaccine ID, and the side effects. Each block transaction in the blockchain would contain a special QR code that cannot be replicated and duplicated for any other vaccine in the future. A similar block structure is there for the distributors blockchain. Distributors have the responsibility of selling the vaccines to the hospitals further. Thus the distribution details also need to be properly maintained.

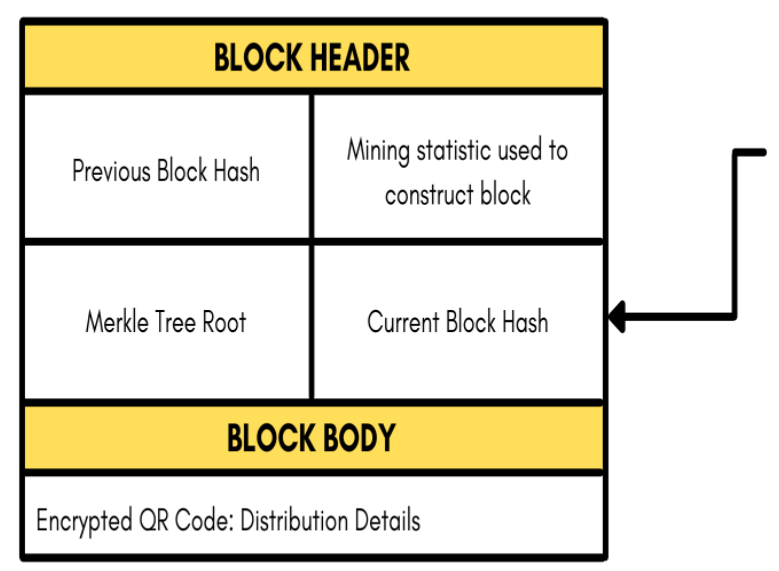

Fig. 5. Block Information for the Distributors Database : consisting of Block Header and Block Body

As shown in Fig.5, the block for the distributors also contains the block header and the block body. Only difference from the manufacturers blockchain comes in the block body where the distributors blockchain contains the encrypted QR code consisting of the distribution information of the vaccine, i.e. distributors information (ID, name, address, etc), and the time- 
stamps of different sub-processes taking place on the vaccine.

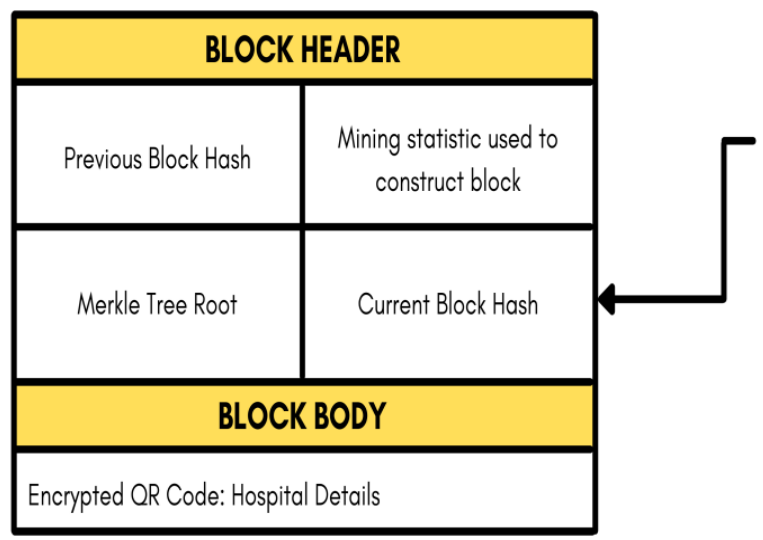

Fig. 6. Block Information for the Hospitals Database : consisting of Block Header and Block Body

And similarly as shown for Fig.6, for the hospitals database or hospitals blockchain, the encrypted QRCode would consist of hospitals information (ID, name, address, etc), status of the vaccine (If the vaccination process is completed using this vaccine or the vaccine was found to be damaged). Thus overall these three private blockchains maintain a record of details different processes accordingly. The use of three different private blockchains instead of one common blockchain has a lot of advantages. Using three different blockchains decreases the load on a single database as if there was a common database for all the entities then there would be a huge number of participants. Using three different blockchains increases the time and overall efficiency to which the blocks are added to the blockchain as well as inquiring the data from them. Also, there is a need for regulation i.e. checking and abiding of all the laws, rules and regulations by manufacturers, distributors, and the hospitals. Also a contract based agreement is required between manufacturers and the distributors for the supply of the vaccines. This problem is achieved by smart contracts. Smart contracts are lines of code or a computer program that are stored on a blockchain and when the predefined and predetermined conditions are met, they get executed and operated automatically without any form of manual intervention. This largely solves the issues of any form of conflicts between the three entities. Thus there need to a smart contract between the manufacturers and distributors in accordance with the government authorities which would be used to regulate the vaccines automatically and also act as a legal contract between them.

\subsection{Hybrid Blockchain for Requests by Patients for Vaccine Data}

Private blockchain solves the issue of internal management of the databases respectively but there needs to be blockchain which would facilitate all the patients for inquiring about the vaccines and its details. Thus as shown in Fig.7, a hybrid blockchain would be a right fit here.

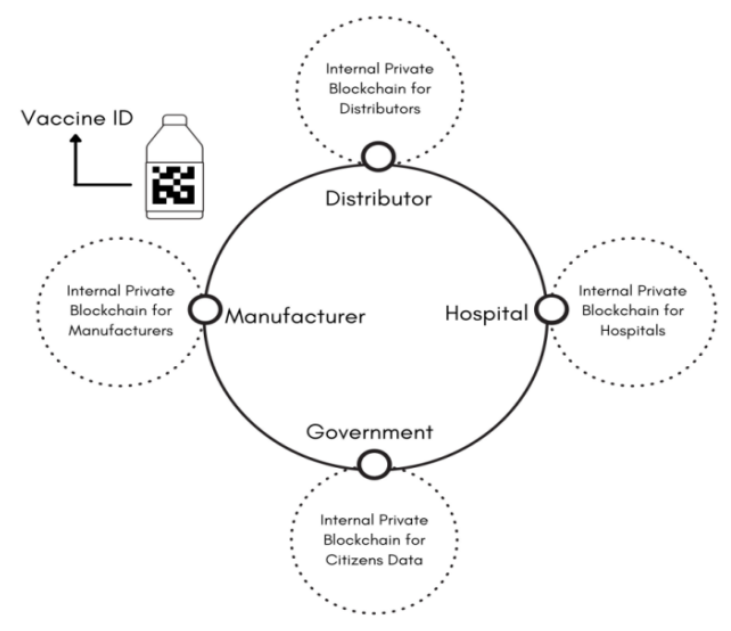

Fig. 7. Hybrid Blockchain (Private Blockchain consisting of several Private Blockchains) for Maintaining the Database for all the Requests by Patients inquiring about their Vaccine Data 


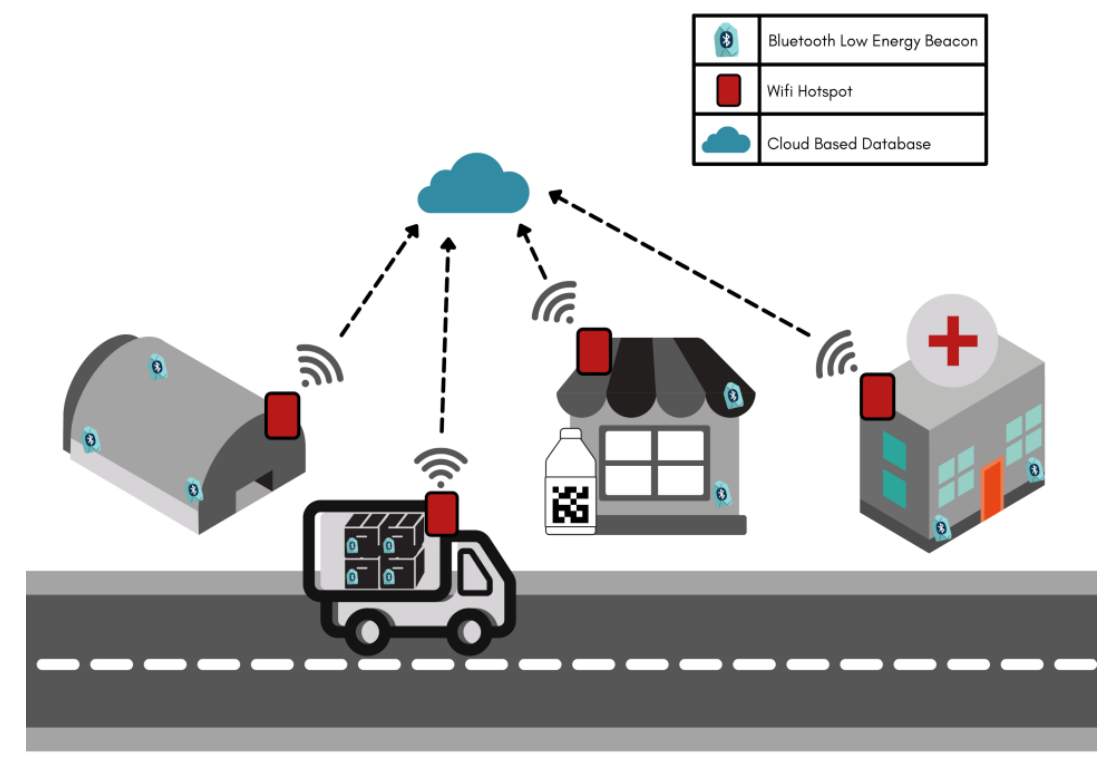

Fig. 8. Visual Representation of the proposed BLE Beacons Network System for Monitoring

In this blockchain, only the manufacturers, distributors, hospitals, and the government authorities could take part. To be specific, not every node from the individual private blockchain would be a part but only specific nodes from the internal private blockchains would be a part. Internal private blockchain of manufacturers, distributors, and the hospitals can then choose to share any amount of their encrypted data with other peers over hybrid blockchain which is externally a private blockchain, according to some access-control policies. The internal communication between the manufacturers, distributors, and hospitals is only through an encrypted QR Code on the vaccine bottle i.e. there is no other communication between them except for the bottle of vaccine which has a QR Code on it which represents its vaccine ID. This type of architecture would help the patients to inquire about the vaccine that they are taking. If any patient wants details of the vaccines, then they would contact the government authority asking for the details while the government would firstly check the patient details and would verify it through the citizens database. If the data of the patient is matched, then the government authorities are responsible for communicating with the manufacturers, distributors and the hospitals asking them for the details of the vaccine by sharing their public key to them. Each of the manufacturer, distributor, and the hospitals have a database on blockchain which has encrypted QR codes data which represent the details of the different processes. Manufacturers, Distributors and Hospitals will then encrypt the QR codes using the public key of the government person who requested for the details and will send the QR codes to it. The QR code can then be decrypted by the only that government node which had requested for the details by using their private keys. After the QR Codes are decrypted, the patients can be informed about the details of the vaccine.

This type of architecture is very effective compared to a public blockchain architecture as the patients don't need to be a part of the blockchain for accessing their details, which immensely reduces the load on the blockchain and getting the information within no time to the patients. If it would have been a public blockchain where any patient could join in, the blockchain would be heavily loaded with so many participants, increasing its cost to a large extent and even decreasing its utility. Inquiries about the vaccine is not a common affair as they already go through a lot of verification before coming in the hands of the patients. Thus there is no need to include patients into the blockchain. The government authority nodes play the role of request-agents and deliver them data effectively. The next challenge that the supply chain management faces is the need for constant monitoring of the vaccines right from manufacturing to the injection of vaccines in the patients. This problem can be dealt by using a network of BLE Beacons. The various ways in which BLE Beacons are discussed further.

\subsection{Use of BLE Beacons for monitoring}

Monitoring the vaccines is a big challenge for supply chain. It involves a lot of manpower which is not effective and turns out to be inaccurate. Using BLE Bluetooth Beacons for tracking the vaccines, their environment, and providing their real time updates to the caretakers can solve this issue. The system described in Fig.8 represents three entities of the supply chain i.e. manufacturers (warehouse), distributors and the hospitals. Now as shown in Fig.8, the system consists of firstly a manufacturer outlet inside which several small devices i.e. Bluetooth Beacons are placed which constantly monitor their areas of proximity (generally up to 20-30 meters) for the vaccine safety. They also keep a check for the workers and their safety constantly. BLE Beacon system consists of three elements which are as follows :

\subsubsection{Bluetooth Low Energy Beacon}

These are the hardware transmitter devices that broadcast signals by tracking their surroundings. 


\subsubsection{WiFi Hotspot}

A hotspot is a geographic location where people can reach the Internet through a wireless local-area network (WLAN). By using WiFi technology, a router is linked to an Internet Service Provider. This connects the BLE Beacons to the internet for the Cloud Database to send the recorded data.

\subsubsection{Cloud Based Database}

This is the entire database for the recorded data which receives data from the BLE Beacons through the Internet and then analyses the data collected to act accordingly in case of any altercation in the behaviour of the surroundings and the vaccines.

As shown in Fig. 8, there are some beacons planted in the manufacturing site (warehouses), and also a WiFi router creates a hotspot for them to connect to the cloud database. The data collected by the beacons includes checking the temperature, pressure, and other parameters of the environment in which the vaccines are stored, the number of boxes of vaccines, any human intervention without further permission, avoiding heavy fork lifting which may lead to damages and checking for all the practices to be followed. Through WiFi network this data is sent to the Cloud Database maintained by the manufacturer company which in turn analyses the data for any kinds of errors or altercations in the behaviour of the vaccines and the environment which might lead to defects.

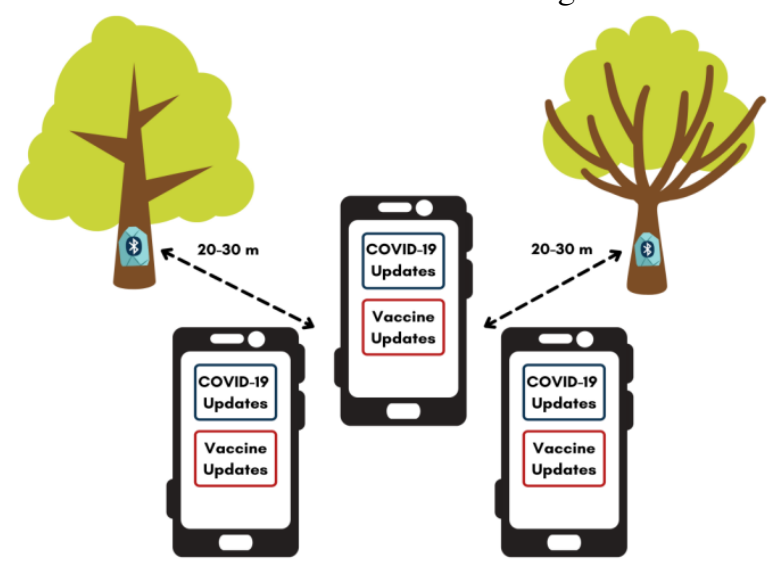

Fig. 9. Visual Representation of the BLE Beacons network set up for transmitting verified information to the Patients

\subsection{Use of BLE Beacons for broadcasting verified information}

BLE Beacons can additionally provide one more set of features which can immensely help in fighting the virus and for smooth conduct of vaccination. As shown in Fig. 9, a network of BLE Beacons can be set up in all the areas in a city to provide verified information to the patients. These beacons can be placed on various objects which are not in touch by human beings in general such as trees. Now any smartphones or Bluetooth Smart enabled device that comes in proximity of these beacons can receive updates regarding the COVID-19 cases in their areas,
Vaccine updates, and can even be informed about the vaccination schedules which has been planned by the government authorities along with the hospitals. This information which is broadcasted would be firstly verified by the authorities and would be received by the beacons to transmit by the cloud database itself. Also these beacons which are placed inside the hospitals would be very helpful for the patients which are coming to get vaccinated for indoor navigation and thus avoiding any types of chaos at the hospitals which could have led to further spread of the virus.

Overall this system is a very effective medium of transmitting verified information to the citizens who could receive numerous updates about the situation of the pandemic, sitting at their homes. They could also be informed about the vaccination schedules and the hospitals where they would get vaccinated.

\section{Conclusion}

The traditional system of using more manpower to monitor and maintaining records of information on a standard database can lead to a lot of errors and chaos especially in context of the vaccines as any kinds of faults or damage in the vaccines can lead to serious health issues in the patients and can even lead to death in the worst case. With the unprecedented rise of Blockchain technology in replacing the traditional databases to incorporate decentralized, tamper-proof databases can largely solve this problem in the present as now the sufficient devices and hardware are also available readily. This could not only help fighting the current pandemic related to COVID-19 but fighting any kinds of diseases in the future. This could be incorporated in the official medical facility structure for general use. At the same time, the use of BLE Beacons can largely solve the issue of monitoring the vaccines and drugs and maintaining their safety. They can also largely solve the issue of spreading of unverified and fake information by broadcasting verified information about various things. These are inexpensive and easy to plant and thus can be incorporated in the current medical facility structure for use. The combination of Blockchain based database and BLE beacons for monitoring looks to be a feasible and effective solution for fighting any diseases and could be included in the current system for general use.

\section{References}

1. WebMD Medical Reference, Coronavirus History. Retrieved from https://www.webmd.com/lung/coronavirus-history (2020, April 15).

2. Schmerker, J. , Covid-19 Testing: A Brief History. Retrieved from https://eu.idtdna.com/pages/community/blog/post/c ovid-19-testing-a-brief-history (2020, May 30).

3. World Health Organization, 172 countries and multiple candidate vaccines engaged in COVID-19 vaccine Global Access Facility. Retrieved from 
https://www.who.int/news/item/24-08-2020-172countries-and-multiple-candidate-vaccinesengaged-in-covid-19-vaccine-global-access-facility (2020, August 24)

4. United Nations News, WHO presses for greater investment in global COVID-19 vaccine facility. Retrieved from https://news.un.org/en/story/2020/08/1070912 (2020, August 24)

5. Craven, J., COVID-19 vaccine tracker. Retrieved from $\mathrm{https}: / / \mathrm{www}$.raps.org/news-and-articles/newsarticles/2020/3/covid-19-vaccine-tracker $\quad$ (2020, December 23).

6. Pfizer-BioNTech, Covid-19 Vaccine U.S. Distribution Fact Sheet. Retrieved from https://www.pfizer.com/news/hottopics/covid_19 vaccine u s distribution fact she et (2020, November 20).

7. World Health Organization, Growing threat from counterfeit medicines. Retrieved from https://www.who.int/bulletin/volumes/88/4/10020410/en/ (2010, April)

8. Ali, Muhammad Salek \& Vecchio, Massimo \& Pincheira Caro, Miguel Rodrigo \& Dolui, Koustabh \& Antonelli, Fabio \& Rehmani, Mubashir Husain. (2018), Applications of Blockchains in the Internet of Things: A Comprehensive Survey, IEEE Communications Surveys \& Tutorials. PP. 1-1. 10.1109/COMST.2018.2886932. 